Document downloaded from:

http://hdl.handle.net/10251/154512

This paper must be cited as:

Sánchez-Marín, JR.; Bachiller Martin, MC.; Nova-Giménez, V.; Boria Esbert, VE. (2019). Reconfigurable Resonator in Decoupled Empty SIW Technology Using Liquid Crystal Material. Electronics Letters. 55(16):907-910. https://doi.org/10.1049/el.2019.1088

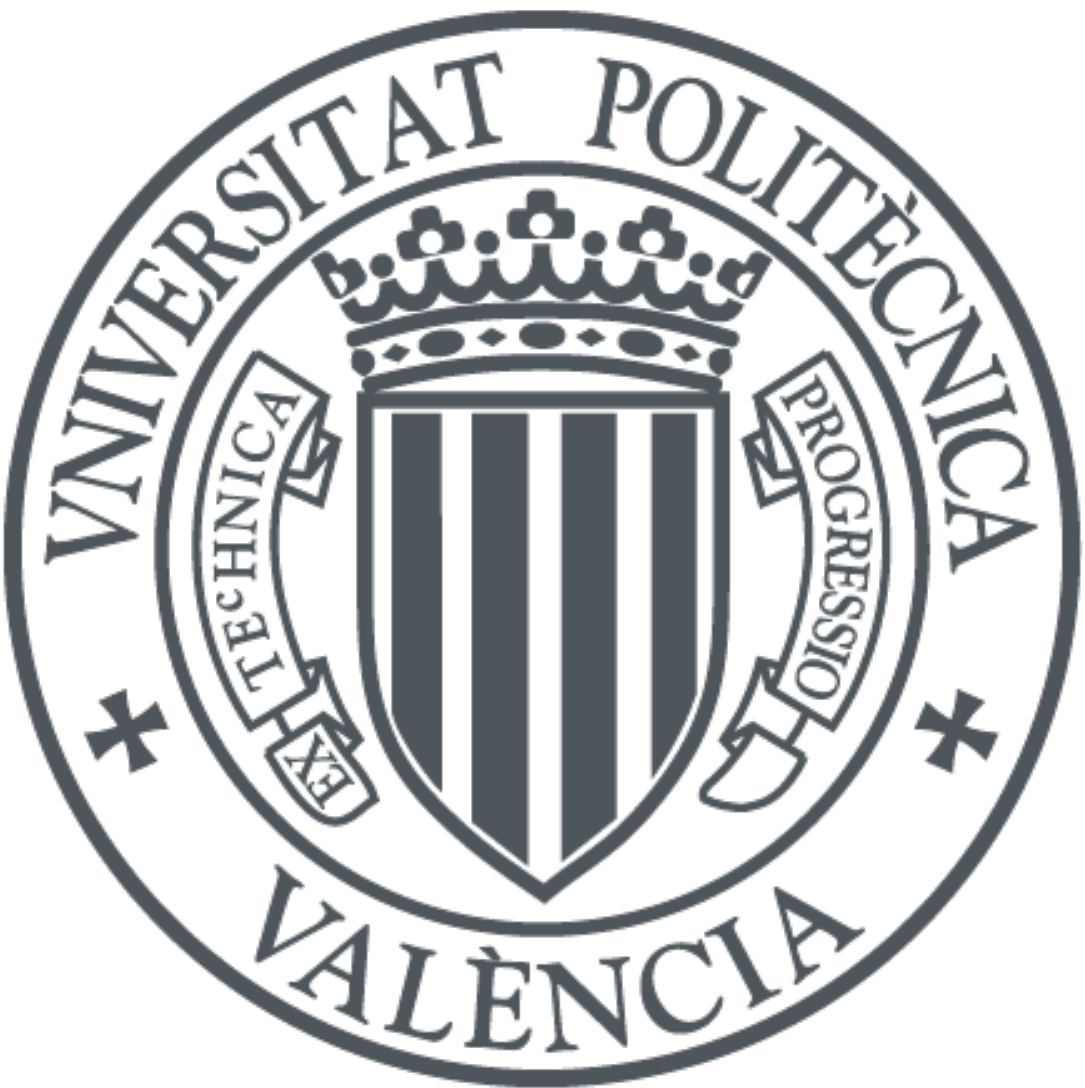

The final publication is available at

https://doi.org/10.1049/el.2019.1088

Copyright Institution of Electrical Engineers

Additional Information

"This paper is a postprint of a paper submitted to and accepted for publication in Electronics Letters and is subject to Institution of Engineering and Technology Copyright. The copy of record is available at IET Digital Library" 


\section{Reconfigurable Resonator in Decoupled Empty SiW Technology Using Liquid Crystal Material}

\author{
Juan R. Sánchez, Carmen Bachiller, Vicente Nova, and Vicente
} E. Boria

This paper presents a novel continuously tunable microwave resonator based on Liquid Crystal (LC) material. The reconfigurable device is implemented in Decoupled Empty Substrate Integrated Waveguide (DESIW) technology. DESIW technology allows both magnetic and electric DC (or low frequency) biasing of the LC. The resonator is continuously tunable in a range of $600 \mathrm{MHz}$, resulting in a measured tunability range of $8.5 \%$ around a centre frequency of $7 \mathrm{GHz}$. Moreover, the measured insertion loss of the reconfigurable device is between $4.3 \mathrm{~dB}$ and $6.8 \mathrm{~dB}$, thus obtaining a Q-factor of 190-172.

Introduction: One of the current needs in high-frequency components for new communication systems is their capability to provide adaptive frequency responses. This reconfigurable behaviour is required because of the increasing spectrum congestion and the demand for keeping a constant high information flow in many different scenarios. Resonators (as basic building blocks of many other passive components, mainly filters for instance) are key elements in any RF front-end of communication and detection systems, so they are perfect candidates where implementing reconfiguration features.

There are different options to fulfill a frequency reconfigurable performance. Mechanical elements have been traditionally used and, more recently, tiny pieces with electromechanical movement (RF MEMS) [1], or elements driven by an electric current as varactors, PIN diodes or ferrites $[2,3]$ have been introduced. These elements can usually perform a digital response with a few discrete levels.

In recent years, liquid crystals (LCs) have been used to achieve this reconfigurable performance $[4,5]$. These materials have a variable degree of dielectric anisotropy, which means that the value of their electric permittivity can be modified at will due to some stimulus, such as a very low frequency electromagnetic field. The use of new liquid crystal mixtures, with high anisotropy range and low loss, opens a wide and promising field of possibilities for providing an analogical (continuous) control of reconfiguration levels.

The goal of this work is to develop a continuous reconfigurable resonator with high tunability range. This new device is based on Decoupled Empty Substrate Integrated Waveguide (DESIW) [6] technology filled with a tunable material (LC). Since DESIW is a decoupled structure, an electromagnetic bias field can be applied in order to polarize the LC molecules.

Liquid Crystal technology: LC is an anisotropic liquid material that presents an intermediate matter state with characteristics between crystalline solids and isotropic liquids, called mesophase. In this state, the LC mixtures present a series of properties of molecular mobility and dielectric anisotropy. These properties allow that the dielectric permittivity of the LC is adjusted within a range of continuous values by using an electric signal.

The rod-like shape of the molecules of LC enables the different values of permittivity depending on their orientation. In the macroscopic scale, the direction of the molecules is represented by a vector called director $(\vec{n})$, which is parallel to the longitudinal axis of the LC molecules. The dielectric permittivity values of the nematic LCs are defined by the angle formed between the director vector and an external RF electric field $\vec{E}$, as shown in Fig. 1.

On the one hand, the propagation of an RF signal, which electric field $\vec{E}$ is orthogonal to the long axis of the molecules, will result in the lowest extreme permittivity, i.e. orthogonal permittivity $\varepsilon_{r \perp}$. On the other hand, an RF signal with a polarization of the electric field $\vec{E}$ parallel to the long axis of the molecules will result in the highest permittivity value, i.e. the parallel permittivity $\varepsilon_{r} \|$. Any change in the relative direction between the RF electric field and the molecules axis will result in an intermediate permittivity value. Taking into account this behaviour, the dielectric anisotropy of the material is defined as follows:

$$
\Delta \varepsilon_{r}=\varepsilon_{r \|}-\varepsilon_{r \perp}
$$

The $\varepsilon_{r \perp}$ state is achieved by using an anchoring force (physical alignment) or an electromagnetic DC (or low frequency) bias field, while the $\varepsilon_{r \|}$ state is achieved only by applying the bias field [7].

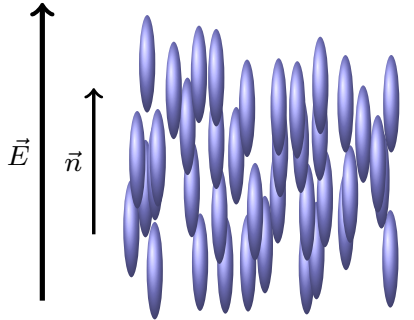

(a)

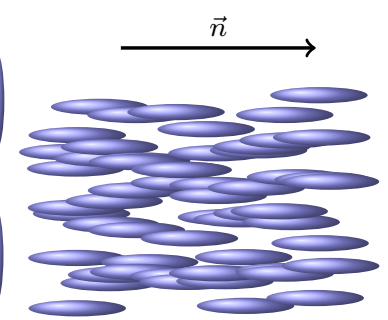

(b)
Fig. 1 Aligned LC molecules showing the director vector $\vec{n}$ and the electric field $\vec{E}$. (a) Parallel permittivity ; (b) Perpendicular permittivity

Resonator topology: The basic idea of the DESIW line is to maintain the three layered structure of the Empty-SIW (ESIW) techonology [8], but decoupling one of the metallic covers. For the fundamental mode of the waveguide, the RF electric field currents flow on the surface of the structure. If DC decoupling is pursued, the continuity of these high frequency surface currents should be granted. To avoid cutting the currents and inducing radiation, a square pattern grid is craved on the surface of the top cover, i.e. a cover divided in several isolated metal squares. Thus, RF currents can flow through the grid created between the squares, preventing interruption by the discontinuity as shown in Figure 2.

The design of this square grid surface, also called Frequency Selective Surface (FSS) [9] cannot be random. FSSs have a passband response in a frequency range, so this behaviour must be far from the working frequency band of the transmission line. This will assure the transmission together with an AC/DC decoupling.

Munk [9] states that the resonance frequency of a FSS (formed by loaded square apertures with a solid plate inside as in this case), has its resonance frequency at $f_{r}=c / \lambda_{r}$, being $c$ the speed of the light and $\lambda_{r} / 4$ the side size of the aperture. The apertures must be designed small enough to have the resonance frequency out of the desired working band. Therefore, the cover with the FSS cells will have a reflection parameter high enough to ensure that the entire structure behaves like a conventional ESIW.

The voltage can be applied between each small inner square and the rest of the structure. It is expected that this solution, even considering some low radiation loss, has a proper performance. In order to have access to the decoupled conductors from outside of the structure, it is needed to drill a set of metallized vias in the center of each square, between the bottom and top sides of the upper cover of the DESIW. In this way, the bias voltage can be applied to the surface of the waveguide. Moreover, different bias voltages could be applied to different sections of the waveguide, since the squares are accessible separately.

The DESIW resonator is designed to be centred at $6.5 \mathrm{GHz}$ when it is filled with a material of permittivity $\varepsilon_{r}=3$. For the designing process, the basic equations of waveguide microwave filters [10] are used, and the transition is based on the solution proposed in [11], obtaining the dimensions shown in Table 1. Afterwards, the prototype is manufactured using a standard milling machine technique [8].

Table 1: Dimensions of the filled DESIW resonator

\begin{tabular}{|l|l|l|l|}
\hline Parameter & Dimension $(\mathrm{mm})$ & Parameter & Dimension $(\mathrm{mm})$ \\
\hline$a$ & 19.050 & $c_{a}$ & 1.336 \\
\hline$a_{\text {win }}$ & 8.000 & $c_{l i n}$ & 0.918 \\
\hline$l_{\text {in }}$ & 12.000 & $c_{l w}$ & 0.700 \\
\hline$l_{\text {win }}$ & 1.000 & $c_{l r}$ & 1.217 \\
\hline$l_{\text {res }}$ & 15.176 & $d_{a}$ & 0.950 \\
\hline$g$ & 0.150 & $d_{l}$ & 0.670 \\
\hline
\end{tabular}

After manufacturing, and before assembling the three layers, the covers are rubbed with natural silk velvet for creating an anchoring surface of microgrooves, which will help the LC molecules to lay in a given direction. For changing this orientation, an external DC (or low frequency) bias field is necessary, either electric or magnetic. 


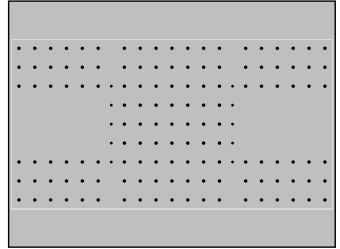

(a)

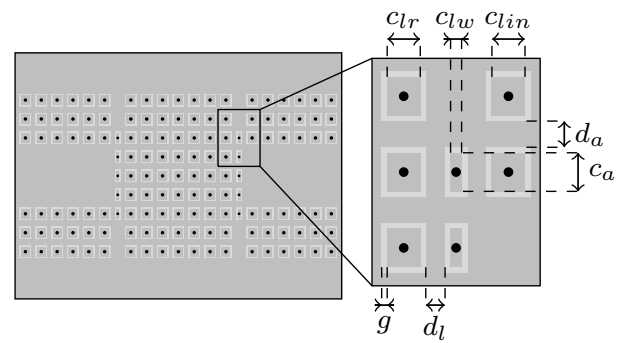

(b)

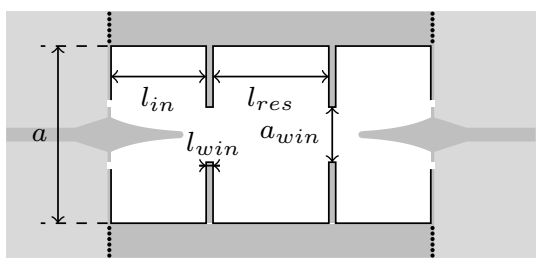

(c)

Fig. 2 Layout of a DESIW resonator with the top cover cut in small squares. In black the metallized vias and the border copper metallization, in dark gray the copper metallization on top of the layer, in light gray the dielectric substrate, and in white the air-filled sections. (a) Top view of the top cover. (b) Bottom view of the top cover, with detail of the squares. (c) Top view of the central body.

\section{Magnetic biasing}

Two magnets can be used for obtaining the two states, since the molecules are aligned parallel to the magnetic field lines. First, the perpendicular permittivity $\left(\varepsilon_{r \perp}\right)$ is achieved by two magnets repelling each other, as shown in Fig. 3(a). Then, the molecules are aligned orthogonally to the RF electric field $(\vec{E})$. Next, the molecules can be aligned vertically, i.e. parallel to the same RF electric field $\left(\varepsilon_{r \|}\right)$, if the two magnets are placed in attracting mode, as shown in Fig. 3(b). When the device is placed between the two magnets, the perpendicular and the parallel LC states can be alternatively measured, by changing the position of the magnets.

\section{Electric biasing}

For electric biasing, a low frequency voltage is applied between the grid of squares and the rest of the structure. When no voltage is applied, the molecules are in repose state, forced by the anchoring surface, obtaining a pseudo-perpendicular permittivity value. When starting to apply a voltage higher than a threshold value (which is different for each LC mixture) [7], the molecules start rotating to the vertical position. Depending on the applied voltage, continuous values of permittivity can be obtained, until $\varepsilon_{r \|}$ is achieved.

Results: In order to test the variation of the frequency response, a DESIW resonator (being part of a 1-pole band-pass filter) is manufactured using RO4003C substrate with $\varepsilon_{r}=3.55,0.813 \mathrm{~mm}$ of height and $17.5 \mu \mathrm{m}$ of copper cladding for the three layers. The resonator is filled with QYPDLC036 (Q036) from Qingdao QY Liquid Crystal Co. The main characteristics of this LC mixture at $5 \mathrm{GHz}$ are $\varepsilon_{r \|}=3.08, \tan \delta_{\|}=0.017, \varepsilon_{r \perp}=2.28$, and $\tan \delta_{\perp}=0.075$, as characterized in [12]. This resonator has been measured applying both magnetic and electric bias fields as shown in Fig. 4.

Fig. 5 shows the frequency response for the two states of the magnetic biasing, and the continuous reconfiguration response for the electric biasing.

The repose measurement, without any field, shows a resonance at 6.74 $\mathrm{GHz}$. There is a frequency shift with regard to the simulation data, due

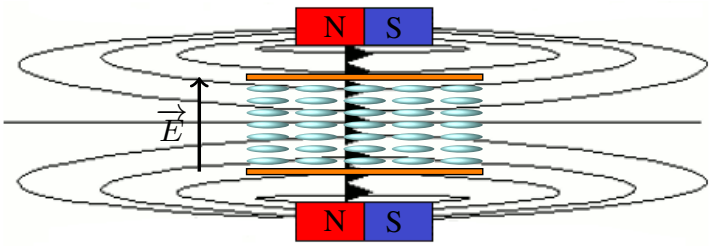

(a) Repel: perpendicular permittivity

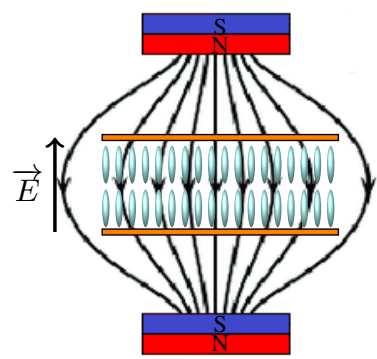

(b) Attract: parallel permittivity

Fig. 3. Cross section of magnetic field biasing states.

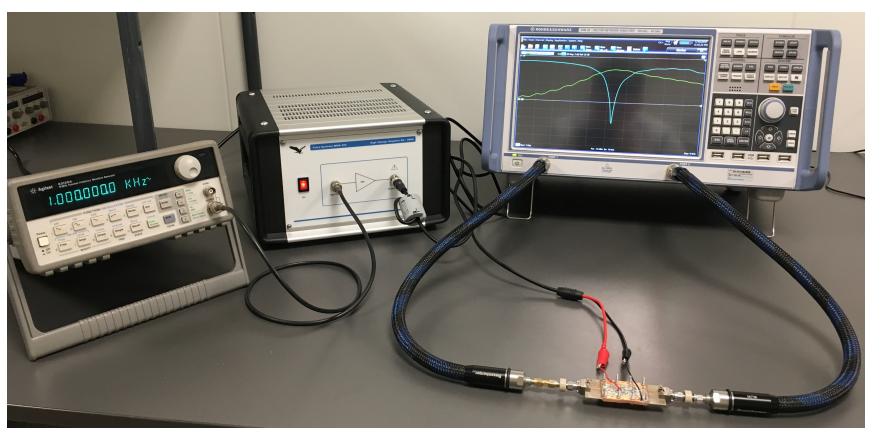

Fig. 4. Measurement set-up using electric bias field.

to the non-perfect perpendicular alignment of the LC, thus decreasing the resonance frequency. When the bias voltage (from $25 \mathrm{Vpp}$ to $100 \mathrm{Vpp}$ in $25 \mathrm{Vpp}$ steps) is applied, the molecules start rotating from the repose state to the parallel position, where the resonance frequency is located at 6.52 $\mathrm{GHz}$. The tuning achieved is continuous in a frequency range of $220 \mathrm{MHz}$, which in relative terms corresponds to $3.38 \%$.

Alternatively, a magnetic biasing field is applied, as shown in Fig. 3. For the perpendicular mode, the resonance frequency is $6.85 \mathrm{GHz}$, and for the parallel one it is $6.3 \mathrm{GHz}$. This tuning range of $550 \mathrm{MHz}$ corresponds to a relative value of $8.5 \%$ that compares rather well with the simulated value of $11 \%$.

The magnetic biasing achieves wider tuning range than the electric one. There are two main reasons for such results. For the parallel case, the voltage is not applied between the whole top cover and the bottom cover, but between the squares and the bottom cover, leaving some spaces without polarization. For the perpendicular case, non electric field can be applied, so the alignment is limited to the repose state configuration. In the magnetic case, the polarization is applied in the whole surface of the device.

Furthermore, the measured insertion loss are between $5.7 \mathrm{~dB}$ and 7.4 $\mathrm{dB}$ in the passband over the whole tuning range, including the losses of both transitions and connectors. The extracted unloaded quality factor $Q_{u}$ ranges between 80 to 30 . These results are mostly determined by the LC properties.

For this reason, another LC mixture has been used, GT3-23002 (GT3) from Merck $\mathrm{KGaA}$. The main characteristics of this LC mixture at 5 $\mathrm{GHz}$ are $\varepsilon_{r \|}=2.59, \tan \delta_{\|}=0.005, \varepsilon_{r \perp}=1.94$, and $\tan \delta_{\perp}=0.039$, as characterized in [12]. In this case, only the magnetic biasing is applied, as shown in Fig. 6. The reason for using only this biasing is to compare the measurement with the simulated values, which use the extreme dielectric permittivity values extracted from the characterization of the mixture.

The repose measurement shows a resonance at $7.27 \mathrm{GHz}$. As in the previous case, there is a shift with regard the simulation due to the nonperfect initial alignment of the LC molecules. For the perpendicular case, 

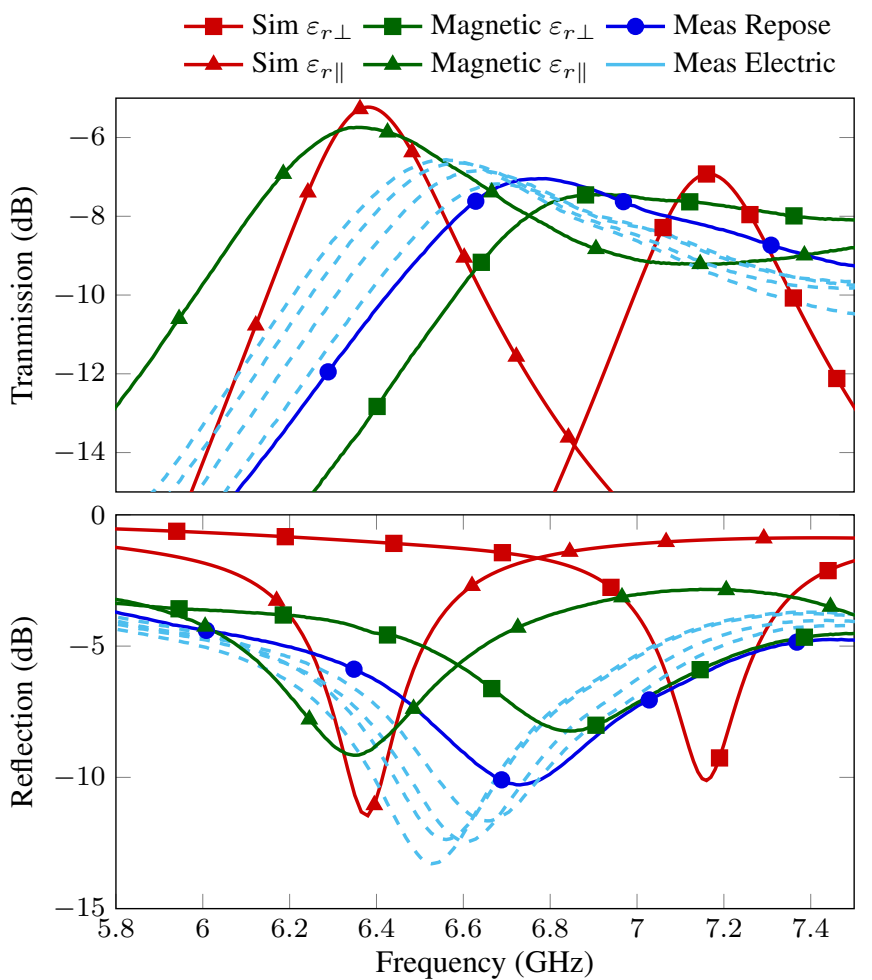

Fig. 5 Comparison between simulated and measured frequency responses for biased DESIW resonator.

the resonance frequency is $7.3 \mathrm{GHz}$, and for the parallel one it is $6.7 \mathrm{GHz}$. This tuning range of $600 \mathrm{MHz}$ corresponds to a relative value of $8.5 \%$ that compares rather well with the simulated value of $13.85 \%$. The measured insertion loss is between $4.3 \mathrm{~dB}$ and $6.8 \mathrm{~dB}$ in the passband over the whole tuning range, including the loss of both transitions and connectors. The extracted unloaded quality factor $Q_{u}$ ranges between 190 and 172 .

For a fair comparison with other works, the LC mixtures should be the same one. However, a comparison with other tunable resonators using LC material is done in Table 2. The use of other LC mixtures can change both the tunabilitiy range and the insertion loss of the proposed structure as demonstrated in this work.

Table 2: Comparison of tunable LC resonators on planar technology

\begin{tabular}{|l|l|l|l|l|}
\hline Work & $\mathrm{f}(\mathrm{GHz})$ & Tuning $(\%)$ & Loss $(\mathrm{dB})$ & $Q_{u}$-factor \\
\hline DESIW Q036 & 6.5 & 8.50 & $5.7-7.4$ & $80-30$ \\
\hline DESIW GT3 & 7.0 & 8.50 & $4.3-6.8$ & $190-172$ \\
\hline Waveguide [13] & 23.5 & 2.10 & Not given & $256-170$ \\
\hline SIW [4] & 23.5 & 1.75 & $27-28$ & $105-102$ \\
\hline Microstrip [5] & 2.8 & 8.20 & $0.97-1$ & Not given \\
\hline
\end{tabular}

Conclusion: A fully continuous tunable resonator has been designed and manufactured in this work. For achieving this wide tunability range, a combination of both a magnetic and electric biasing fields can be used. The magnetic biasing allows to get the extreme states, whereas the electric biasing achieves the continuous intermediate states.

Acknowledgment: This work was partially funded by the Generalitat Valenciana research project PROMETEOII/2015/005, by the Ministerio de Educación, Cultura y Deporte (Spain) under the Fellowship Program for Training University Professors FPU14/00150, and by Ministerio de Economía y Competitividad (Spain) under R\&D project TEC2016-75934-C4-1-R.

Juan R. Sánchez, Carmen Bachiller, Vicente Nova, and Vicente E. Boria (Instituto de Telecomunicaciones y Aplicaciones Multimedia, Universitat Politecnica de Valencia, 46022 Valencia, Spain).

E-mail: juasncm1@upv.es; mabacmar@dcom.upv.es

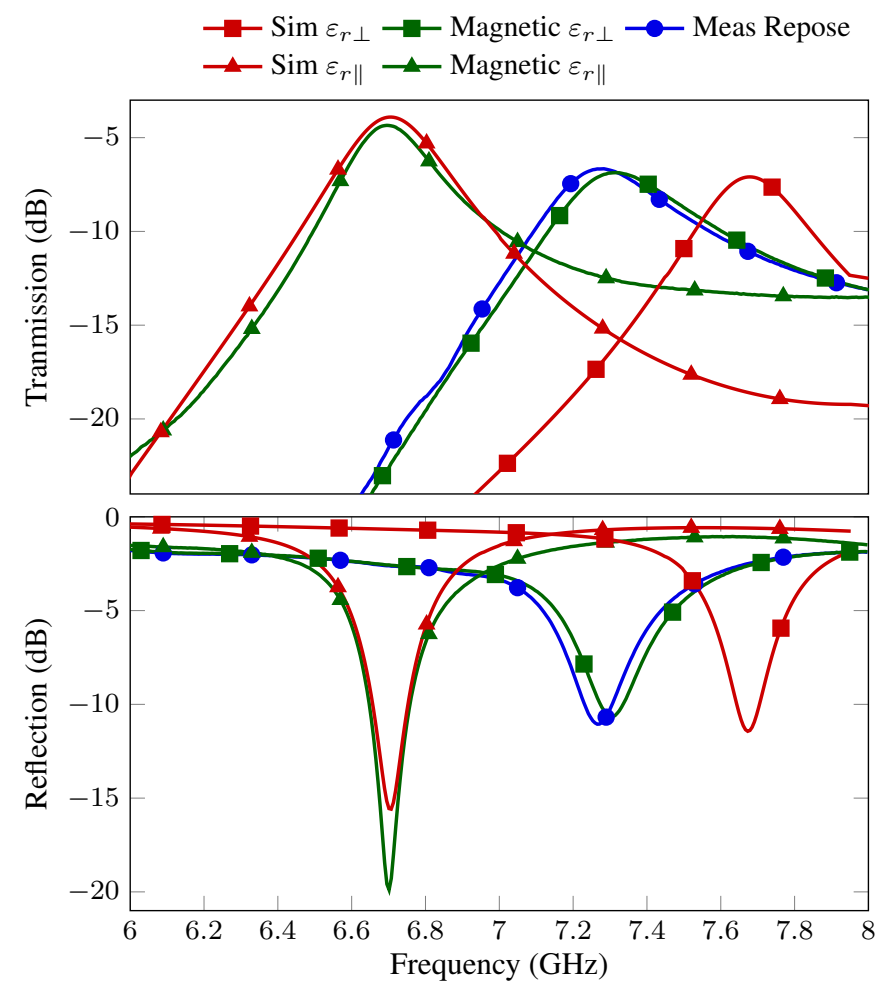

Fig. 6 Comparison between simulated and measured frequency responses for biased DESIW resonator.

\section{References}

1 V. Sekar, M. Armendariz, and K. Entesari, "A 1.2-1.6 GHz SubstrateIntegrated-Waveguide RF MEMS tunable filter," IEEE Trans. Microw. Theory Techn., vol. 59, no. 4, pp. 866-876, April 2011.

2 M. Armendariz, V. Sekar, and K. Entesari, "Tunable SIW bandpass filters with PIN diodes," in Eur. Microw. Conf. (EuMC), 2010.

3 S. Adhikari, Y. Ban, and K. Wu, "Magnetically tunable ferrite loaded substrate integrated waveguide cavity resonator," IEEE Microw. Compon. Lett., vol. 21, no. 3, pp. 139-141, March 2011.

4 A. E. Prasetiadi, O. H. Karabey, C. Weickhmann, T. Franke, W. Hu, M. Jost, M. Nickel, and R. Jakoby, "Continuously tunable substrate integrated waveguide bandpass filter in liquid crystal technology with magnetic biasing," Electron. Lett., vol. 51, no. 20, pp. 1584-1585, 2015.

5 P. Yaghmaee, C. Fumeaux, B. Bates, A. Manabe, O. H. Karabey, and R. Jakoby, "Frequency tunable S-band resonator using nematic liquid crystal," Electron. Lett., vol. 48, no. 13, pp. 798-800, June 2012.

6 J. R. Sanchez, C. Bachiller, H. Esteban, A. Belenguer, V. Nova, and V. Boria, "New decoupled empty substrate integrated waveguide realisation," Electron. Lett., vol. 53, no. 17, pp. 1203-1205, 2017.

7 D. Yang and S. Wu, Fundamentals of Liquid Crystal Devices. West Sussex, England: John Wiley \& Sons, Ltd., 2006.

8 A. Belenguer, H. Esteban, and V. E. Boria, "Novel empty substrate integrated waveguide for high performance microwave integrated circuits," IEEE Trans. Microw. Theory Techn., vol. 62, no. 4, pp. 832839, 2014.

9 B. A. Munk, Frequency Selective Surfaces. Theory and Design. New York, N. Y.: John Wiley and Sons, Inc., 2000.

10 G. Matthaei, E. Jones, and L. Young, Microwave Filters ImpedanceMatching Networks, and Coupling Structures. Artech House, Feb., 1980.

11 H. Esteban, A. Belenguer, J. R. Sanchez, C. Bachiller, and V. E. Boria, "Improved low reflection transition from microstrip line to empty substrate-integrated waveguide," IEEE Microw. Compon. Lett., vol. 27, no. 8, pp. 685-687, Aug 2017.

12 J. R. Sanchez, V. Nova, C. Bachiller, B. Villacampa, A. de la Rua, R. Kronberger, F. Penaranda, and V. E. Boria, "Characterization of nematic liquid crystal at microwave frequencies using split-cylinder resonator method," IEEE Trans. Microw. Theory Techn., p. Not yet published, 2019.

13 T. Franke, A. Gaebler, A. E. Prasetiadi, and R. Jakoby, "Tunable Ka-band waveguide resonators and a small band band-pass filter based on liquid crystals," in 2014 44th Eur. Microw. Conf., Oct 2014, pp. 339-342. 